\title{
Lung Carcinoma Metastatic in the Liver
}

National Cancer Institute

\section{Source}

National Cancer Institute. Lung Carcinoma Metastatic in the Liver. NCI Thesaurus. Code C36306.

A carcinoma that originates from the lung and has spread to the liver. 